\title{
Customized Low-Cost 3D Printed Helmet as a Temporary Measure for a Patient with Acrania
}

\author{
Luis Rene González Lucano 1,2, $\quad$ Francisco Javier de la Peña Brambila ${ }^{1,2}$ Juan Eduardo Pérez ${ }^{1,2}$ \\ Paola Hernández Rosales ${ }^{1,2, \odot}$
}

${ }^{1}$ Tecnologico de Monterrey, Escuela de Medicina y Ciencias de la Salud, Monterrey, Nuevo Leon, Mexico

${ }^{2}$ Department of Plastic and Reconstructive Surgery, Hospital Civil Antiguo "Fray Antonio Alcalde," Jalisco, Mexico

Address for correspondence Paola Hernández Rosales, MD, Avenida General Ramón Corona \#2514 Nuevo México, 45138 Zapopan, Jalisco, México (e-mail: paola.hernandez.rosales@gmail.com).

Indian J Plast Surg 2019;52:252-253

Sir,

3D printing in medicine has become an important tool in the pursuit of alternative measures for various conditions and it has the potential of becoming more useful in the future.

It consists of using materials such as plastic, metal, and resin and depositing them on one another in layers to produce a three-dimensional (3D) object. It has been primarily used in engineering to create prototypes; however, recent advances in printing materials have now been able to produce objects that are comparable with traditionally manufactured items..$^{1,2}$

There are many potential uses for 3D printing in medicine which could have a significant impact in changing the way patients are treated for various conditions in the future. It has already been proved useful in custom prosthetics, dental implants, and eyeglasses, among others. ${ }^{1}$

$3 \mathrm{D}$ printing became essential in the treatment and progress of our 3-year-old female patient with occipital acrania who at the moment is not a candidate for surgery; the neurosurgery team has decided to wait until the patient is older ( 7 years old) to avoid the need of a second procedure due to bone resorption or head growth if a bone allograft or a titanium implant is used, respectively. ${ }^{3-5}$

For our patient, the occipital acrania represented a big obstacle for her during the rehabilitation process because they were afraid of hurting her due to the lack of protection of the whole occipital lobe. Because of this, an individualized helmet was created by 3D printing from a CT scan reconstruction of the same patient.

Computed tomography (CT) allows to generate a 3D reconstruction of images by detecting the radiation that crosses the body tissues through the sequential acquisition of a series of images separated by equal spaces by axial cuts or transverse oriented perpendicular to the axis; saving the images in digital format of image and communication in medicine (DICOM) that allows to generate an interface between the medical team and another image visualization device. ${ }^{6}$ The 3D reconstruction is done through DICOM Osirix (Dicom Viewer, Bernex, Switzerland),a specialized software for image processing, where the two-dimensional images are stacked on the $\mathrm{z}$ axis by the distance of each cut made by the tomograph and thus reconstruct the 3D model of the patient's head with the cranial deformation which will serve as a guide to generate the helmet model (-Fig. 1).

The generated model is exported to the computer-aided design (CAD) software SolidWorks, where the solid is used as a reference to generate the custom structure of the helmet taking as reference the frontal bone, the atlanto-occipital joint, the temporal bone and sphenoid bone. It has holes to allow ventilation and prevent excessive sweating in the patient.

After making the 3D model of the helmet on the solid of the human figure, an extraction or subtraction between pieces is made to make the hollow of the helmet with the anatomical shape of the head, which will allow the helmet to adapt appropriately on the area that needs to be protected ( - Fig. 2 ).

This helmet can be adapted to the growth of her skull; it is made of a flexible photopolymer resin which has great resistance and flexibility that confers a better fit and comfort to the patient.

Thanks to this helmet, she has had a major breakthrough in physical therapy; her therapy is now performed correctly with not so many restrictions thanks to the protection given by the helmet, this helped her advance a lot faster in her motor development, she can now sit by herself and even stand on her feet for a few seconds with help. It is a reliable option for these types of patients as a temporary measure until they become candidates for a definitive cranioplasty.

Orthotic helmets are an alternative temporary measure for patients who cannot have the surgery or have to postpone it for different reasons; they protect the vulnerable published online

September 12, 2019
Dol https://doi.org/

10.1055/s-0039-1696077

ISSN 0970-0358.
(C)2019 Association of Plastic

Surgeons of India
License terms

(1) $\Theta \circledast$ 


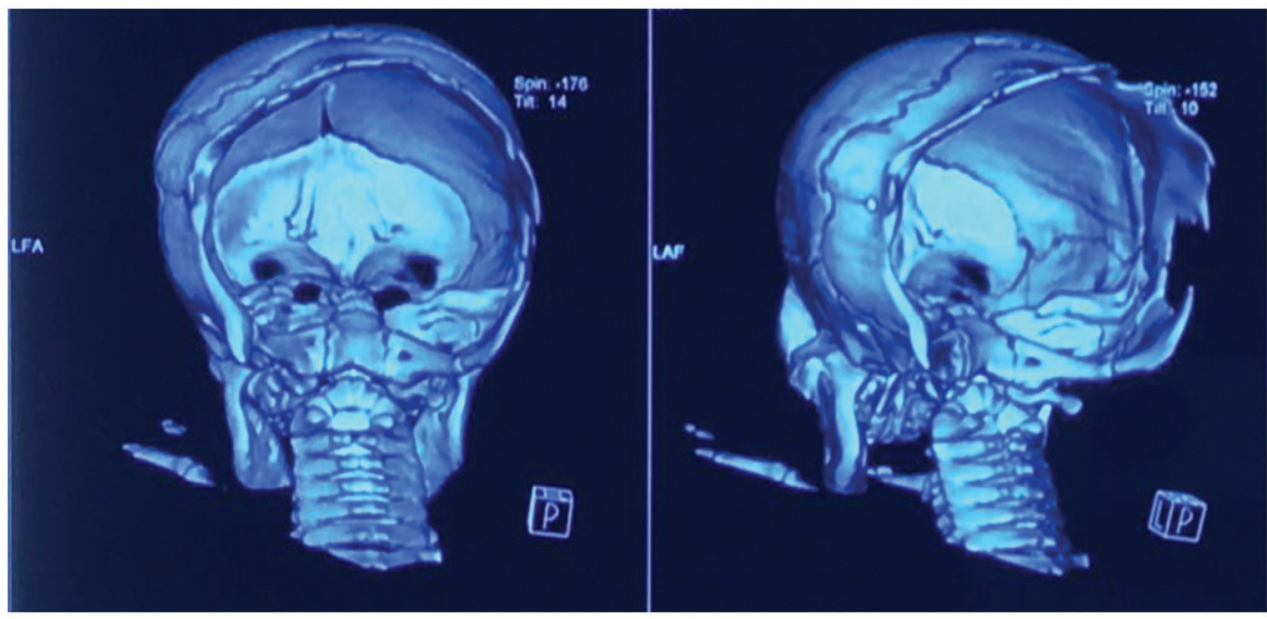

Fig. 1 CT scan 3D model of the patient's head. Posterior and posterolateral view of the defect.
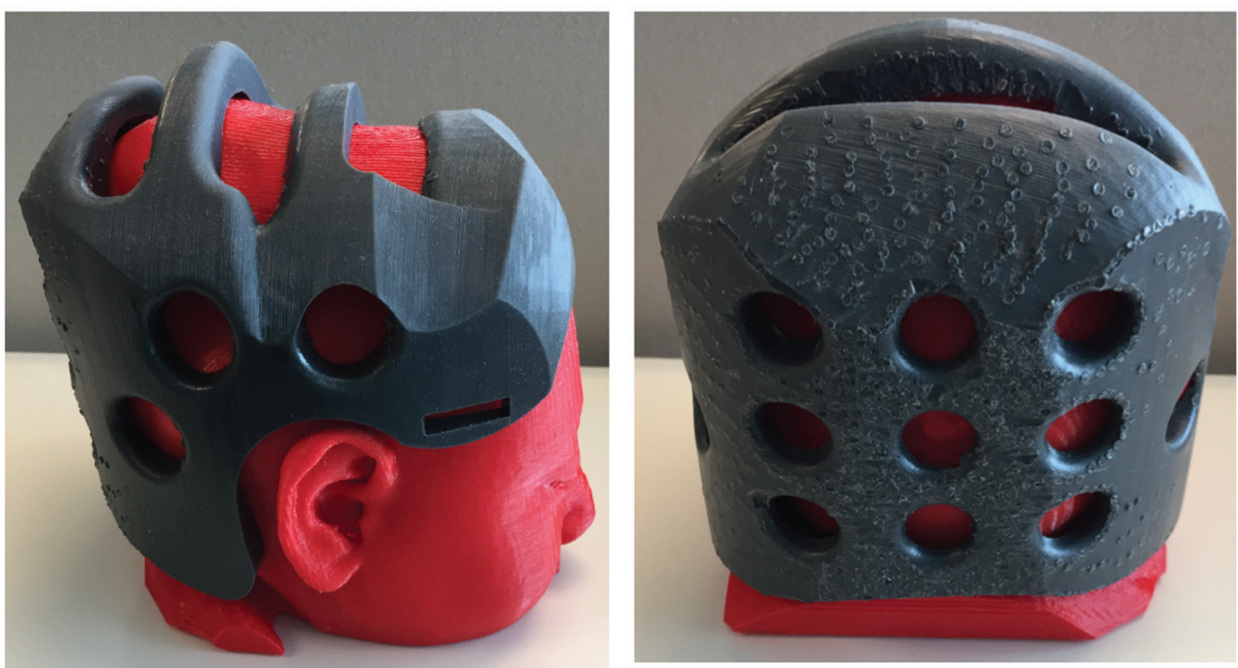

Fig. 2 Final flexible photo polymer resin helmet adapted to the patient's anatomical head shape.

structures and hold them in place until the definitive treatment can be performed. The production of the 3D printed helmet results in an expense of approximately $\$ 200$, while the conventional helmets can reach up to $\$ 1,990$.

These are the reasons that motivated us to propose an alternative low-cost technique for the temporary treatment of patients who have contraindications or do not have the economic resources needed for the surgery or for the conventional orthotic helmets.

By sharing our experience using low-cost 3D printing, we not only prove the success in this particular case, but we also hope to accelerate the pursue in technological research and development to increase the repertoire of options offered for specific pathological entities.

\section{Conflict of Interest}

None.

\section{References}

1 Schubert C, van Langeveld MC, Donoso LA. Innovations in 3D printing: a 3D overview from optics to organs. Br J Ophthalmol 2014;98(2):159-161

2 Gong H, Beauchamp M, Perry S, Woolley AT, Nordin GP. Optical approach to resin formulation for 3D printed microfluidics. RSC Advances 2015;5(129):106621-106632

3 Grant GA, Jolley M, Ellenbogen RG, Roberts TS, Gruss JR, Loeser JD. Failure of autologous bone-assisted cranioplasty following decompressive craniectomy in children and adolescents. J Neurosurg 2004;100(2, Suppl Pediatrics):163-168

4 Williams L, Fan K, Bentley R. Titanium cranioplasty in children and adolescents. J Craniomaxillofac Surg 2016;44(7):789-794

5 Friede $\mathrm{H}$. Normal development and growth of the human neurocranium and cranial base. Scand J. Plast Reconstr Surg 1981;15(3):163-169

6 Hernández Muñiz S. Mitjavila Casanovas M. Introducción a la tomografía computarizada. Rev Esp Med Nucl 2006;25(3):206-219 